RAD Conference Proceedings, vol. 4, pp. 179-184, 2020

ISSN 2466-4626 (online) | DOI: 10.21175/RadProc.2020.36

www.rad-proceedings.org

\title{
SOLAR UV IRRADIATION MONITORING IN BULGARIA USING NARROW-BAND DIGITAL SENSORS AND OPEN-SOURCE INFLUXDB DATABASE
}

\author{
N. Tyutyundzhiev1 ${ }^{*}$, Ch. Angelov ${ }^{2}$, K. Lovchinov3, T. Arsov², H. Nitchev ${ }^{1}$
}

${ }^{1}$ Institute of Electronics, Bulgarian Academy of Sciences, Sofia, Bulgaria

${ }^{2}$ Institute for Nuclear Research and Nuclear Energy, Sofia, Bulgaria

3Institute of Optical Materials and Technologies “Acad. J. Malinowski”, Bulgarian Academy of Sciences, Sofia, Bulgaria

\begin{abstract}
The global climate change in the recent years has increased the interest to atmospheric irradiation monitoring in wider format. Additionally to Gamma-ray and X-ray sensors, $U V-C, U V$-B and $U V$-A sensing systems has been developed to respond to the human expectations about sustainable life and to prevent them from hostile factors. The deeper penetration of UV sensor equipment and dense disperse of monitoring points will improve further the accuracy of measurements, accuracy of forecasting and will convince the citizens in the responsibility of researchers and their work. This work presents UV sensing equipment developed for remote monitoring. Solutions include complex system of narrow band UV sensors, datalogging units, wi-fi communication devices, solar PV charging and Li-Ion energy storing equipment. Most of the components and software technologies are based on opensource platforms and approaches as well as internet-shared results. The analysis of results from $3 \mathrm{UV}$ sensor systems already installed in 3 high-mountain monitoring locations reveals local UV spectral variations. The performance of $U V$ sensors has been evaluated. The collected database during two-year field measurements is prepared for training and development of algorithms for short-term UV forecasting.
\end{abstract}

Keywords: Solar UV measurements, UV sensors, open-source platforms, UV databases

\section{INTRODUCTION}

Solar UV irradiation transmitted through the Earth's atmosphere has a significant effect on the life of the planet. In space, sudden non-regular releases of energy in the solar atmosphere (solar flares) and coronal mass ejections (CMEs) can contribute additionally to so-called ground level effects (GLE) of high-energy particles (protons) bombardments and secondary photons emissions in X-ray and UV regions. On Earth, UV irradiation represents a small portion of the total solar spectrum $(\sim 8 \%)$ but its availability is of great importance because of participation in Ozonegenerating photochemical reactions [1] in the Earth's strato - and troposphere. UV exposure has been linked to skin cancers, corneal damage, immune suppression, erythema and aging of the skin in humans. Apart from its damaging effects in case of human overexposure, UV irradiation can be beneficial. In small doses it initiates the production of vitamin D. Moreover it can be used for disinfection against hostile viruses.

Because of dynamic atmospheric filtering and combined effect of many parameters on the solar activity [2], particle transportation and on-ground UV radiant flux levels, it is difficult to determine the role of each variable separately. It is therefore essential to have as many UV measurements as possible under different environmental conditions (in mountain and urban areas). The current ground-based UV monitoring network does not provide sufficient coverage over wide surface regions but UV records are crucial for validating the indirect (satellites) methods for estimating UV irradiance.

The goal of this study is to present recent success in UV monitoring in the frame of INTERGA_UV project (no. KP 06-24H - 2018 of National Research Fund of Bulgaria) by development of a network of sensors, remote databases and UV monitoring center. Simultaneous 30 sec measurements at UV-B/UVA/Visible/NearIR wavelengths are performed. Daily UV-B/UV-A profiles at different locations are compared and discussed.

\section{UV SENSORS NETWORK}

Remote high-altitude locations are preferable for UV monitoring due to thinner atmospheric layer and fewer gases molecules and aerosols able to scatter or to absorb UV radiation. Other factors are increased surface albedo due to reflecting snow cover and increased secondary photons generated by cosmic rays or solar wind particles. However, because of higher risk of lightning strikes, high winds and low temperatures at these locations it is advisable cost-efficient and lowpower instrumentation to be used.

\footnotetext{
*n_tyut@ie.bas.bg
} 


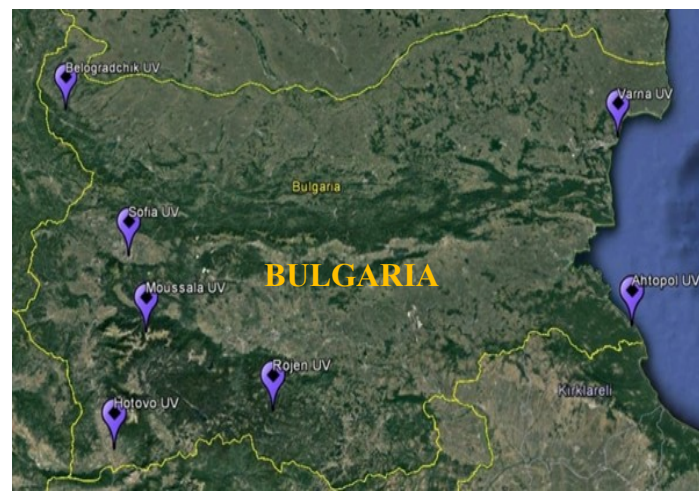

Figure 1. Locations for UV monitoring in Bulgaria

Seven locations have been selected for deployment of UV monitoring equipment, as depicted in Figure 1. All of them are public research areas with developed communication infrastructure. BEO-Moussala is a high-altitude research station situated in Rila mountain at Mount Moussala (2925 m a.s.l.), specialized in monitoring of natural and technogenic radioactivity of atmospheric aerosols. NAO-Rozhen (1759 $\mathrm{m}$ a.s.l.) and NAO-Belogradchik (650 $\mathrm{m}$ a.s.l.) are astronomical observatories situated in areas with high atmospheric transparency. Sofia city location is the most populated and polluted urban area. Hotovo village is the location with highest amount of sunny days in the country. Varna and Ahtopol locations are famous with clean sea-shore environment (0-15 m a.s.l.) and regular coastal winds. Comparisons of solar UV irradiation among the above mentioned locations are a research target in the recent years.

\section{INSTRUMENTS FOR UV-A, UV-B IRRADIATION MEASUREMENTS}

The conventional scanning spectroradiometers provide the highest measurement accuracy but they are slow, costly and require trained personnel for maintenance and operation. Multichannel instruments consist of several filtered semiconductor photodiodes which provide much faster data sampling but being analog devices they need precise calibration. At present, recent advances in UV sensor technology offers narrow-band digital sensors based on visibleblind $\mathrm{SiC}$ or $\mathrm{GaN} / \mathrm{GaAlN}$ structures with integrated A/D converters and low-noise filters at affordable prices. Additional "kick" on sensor system development is the boom of computer systems with an embedded Linux unit, like Arduino, Intel Galileo, Raspberry Pi, Odroid, NVIDIA Nano, whose community is numerous, allowing easier and faster learning of open-source applications and sensor libraries.

The developed UV monitoring system, described elsewhere [3], differs from conventional UV equipment with cost-efficient hardware design and open-source approach concerning database preparation and webbased visualization.

The basic sensor probe consists of two sensor boards:
- a two-channel digital sensor board (VEML 6075 chip), sensitive in UV-A/UV-B ranges with peak sensitivity at UV-A $(365 \mathrm{~nm}$, range $350-375 \mathrm{~nm})$ and UV-B (320nm, range 315-340nm), bandwidth +/- 15 $\mathrm{nm}$.

- a two-channel Visible/nearIR broad-band digital sensor board (TSL2591 chip) with max responsivity centered respectively at $650 \mathrm{~nm}$ and $820 \mathrm{~nm}$ (500$1100 \mathrm{~nm}$ range).

Both sensor boards can be accessed via standard two-wire interchip I2C protocol. At the highest mountain location (BEO-Moussala, 2925m) solar UV-C radiation is expected to penetrated the atmosphere and an additional UV-C sensor is installed (GUVC-T21) with sensitivity range (230-280nm), wavelength peak $=254 \mathrm{~nm}$ ). The developed sensor probes including double quartz bulb encapsulation are presented at Figure 2.

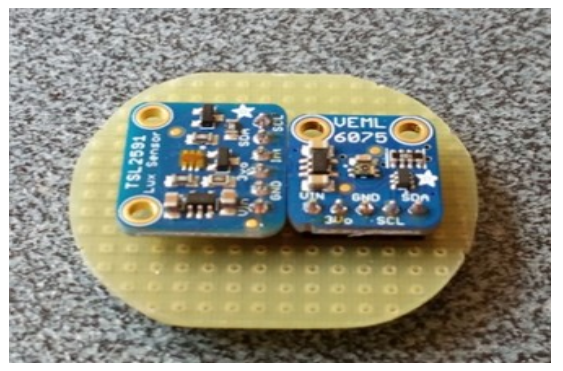

a) VEML 6075, TSL 2591 sensor probe

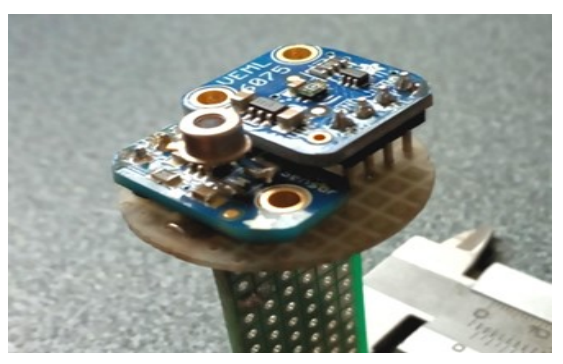

b) VEML 6075, TSL 2591, GUVC-T21 probe

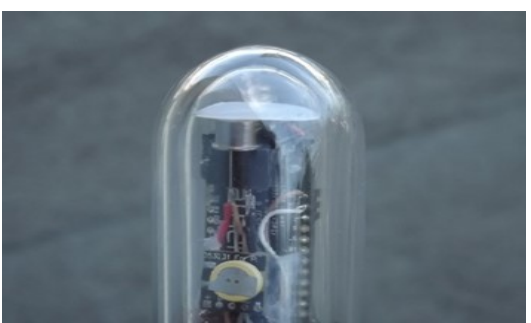

c) double quartz bulb with sensor probe

Figure 2. Experimental sensor probes with digital UV sensors

Additional calibration of every sensor probe has been carried out on-site by comparing the sensor readings to external standardized sensors and applying the correction coefficients to the particular sensor software application. 
The datalogging and acquisition system was developed using open-source hardware - Expressif ESP32 wi-fi microcontroller, a System on a Chip (SoC) Odroid C2 microcomputer (credit-card size) and UBUNTU 18.04 LTS Linux operating system. The databases consist of $30 \mathrm{sec}$ mean values of sensor readings measured on a horizontal surface. Code programming of ESP microcontroller has been performed by $\mathrm{C}++$ application using Arduino IDE environment. The programming environment and the accepted software pipeline for remote monitoring are organized as follows:

$$
\begin{aligned}
& \text { UV sensors => Arduino app on ESP32 => } \\
& \text { =>MQTT broker => InfluxDB => Grafana => } \\
& \text { / Ubuntu LinuxOS on SoC machine / } \\
& =>\text { Central server on Linux Server machine }
\end{aligned}
$$

At first, MQTT Mosquitto broker/server application collects sensor readings over $\mathrm{Wi}-\mathrm{Fi}$ and transports them to the Linux machine. The ESP32 hardware platform is connected as publisher sending open wi-fi messages (sensor readings) to the computer in the same LAN where MQTT broker is running [4]. MQTT protocol is specifically useful for "machine to machine" communication, designed to provide fast transfer of sensor data with a small size. The short distance wi-fi communication was intentionally selected in order to prevent the local databases from damages due to lightning strikes over sensor probes.

Then, python bridging script converts data to the format required by Influxdb database. Influxdb has been selected to store sensor data locally due to its small size, easy data manipulation and useful timeseries format.

Finally, the Influxdb is configured and linked to represent the real-time data using dashboards of Grafana visualization tool [5]. Grafana has been selected for this work due to open-source code, smart graphics and the ability to perform time-series analytics. Grafana is one of the most recent and advanced open-source visualization software tool written in Go language. The output of this software tool, a PNG image, can be seen in a web browser and can be snapshot using remote VNC applications.

As explained above, the developed soft applications are executed on a small Linux machine as server applications in a pipeline sequence. The connection to the central server is implemented using the existing open GSM network and VNC/RDP protocol.

Some illustrative examples of the developed field equipment for remote UV monitoring (UV sensors heads, boxes of electronics with solar chargers) installed at the selected locations are presented at Figure 3 a),b),c).

The PV energy, generated from a $10 \mathrm{Wp}$ solar PV module, charges Li-Ion batteries which are able to provide stand-alone operation during the summer season. Additional UPS devices connected to the LAN are configured to provide continuous operation during the rest of the year.

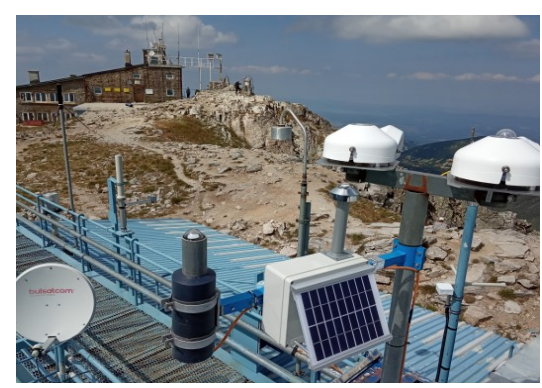

a) BEO-Moussala, $2925 \mathrm{~m}$

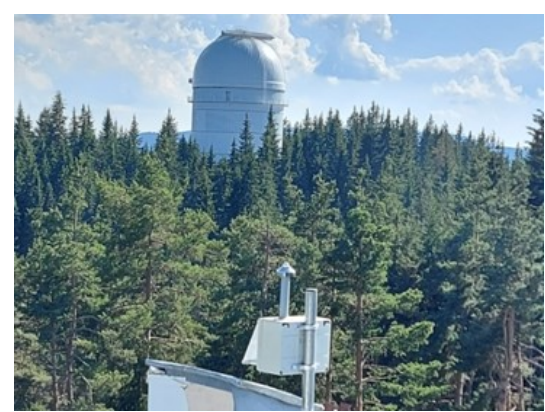

b) NAO-Rozhen, $1759 \mathrm{~m}$

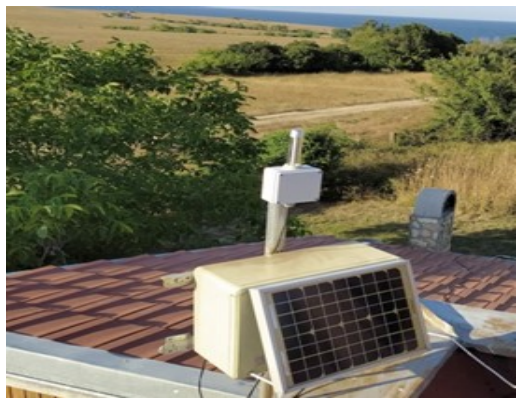

c) HMS-Ahtopol, $12 \mathrm{~m}$

Figure 3. On-field installations of UV sensors equipment

\section{RESULTS AND DisCUSSIONS}

Surface UV levels are strongly affected by clouds, aerosols, altitude, solar zenith angle, and surface albedo. These different factors contribute to high variability in UV radiant fluxes. Additional solar variability occurs as a result of extraterrestrial events sunspots, solar glare, solar eruptions, etc. Because of the combined effect of many parameters on the UV radiant levels, it is difficult to determine the role of each variable separately. It is therefore essential to have many UV measurements under different environmental conditions and different locations.

Summer 2019 and Summer 2020 campaigns were set up to monitor solar global UV-B and UV-A components and their characteristics on selected locations under different atmospheric and climate conditions.

It is reported from other research groups [6] that unexpectedly high UV radiant fluxes occur under partly 
cloudy skies when the sun is not fully obscured. These high-intensity spikes could be explained by additional UV radiant fluxes, result of increased reflection on the edges of dense clouds. As can be seen at Figure 4 below, in some days of high atmospheric transparency at high altitude as mount Moussala, the UV-B flux (dark violet curve) may exceeds UV-A component (light violet).

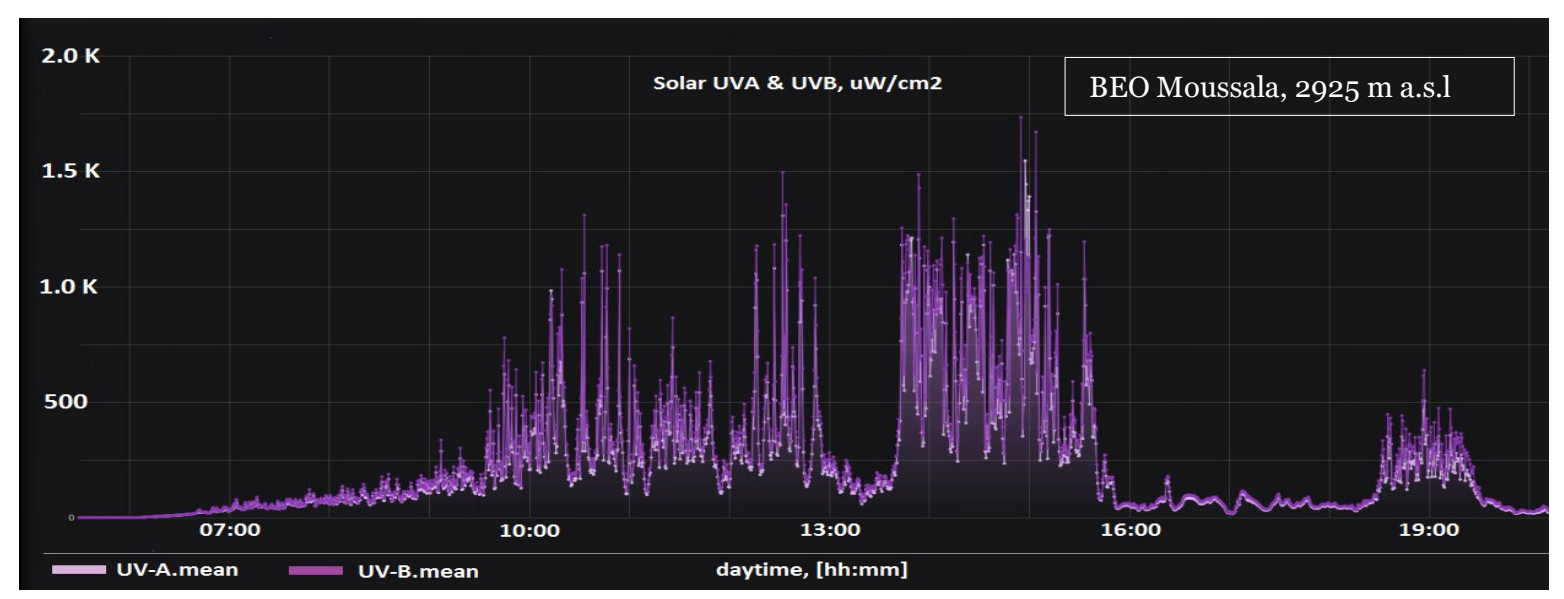

Figure 4. UV-A/UV-B daily profiles at high altitude of mount Moussala on o1 August 2020

Speaking about the intensity of peaks and repetition they could be attributed not only from increased reflection from dense clouds but also to occasional extraterrestrial events of solar activity [7], visible at high altitudes.

\section{- $\quad$ Daily UV attenuation due to pollutants}

The second observable effect on the UV radiant fluxes is associated with atmospheric turbidity-aerosol loadings, as depicted at Figure 5 and Figure 6. Results suggest that the day-to-day variation in pollutants may be a resultant factor producing further UV light scattering and attenuation.

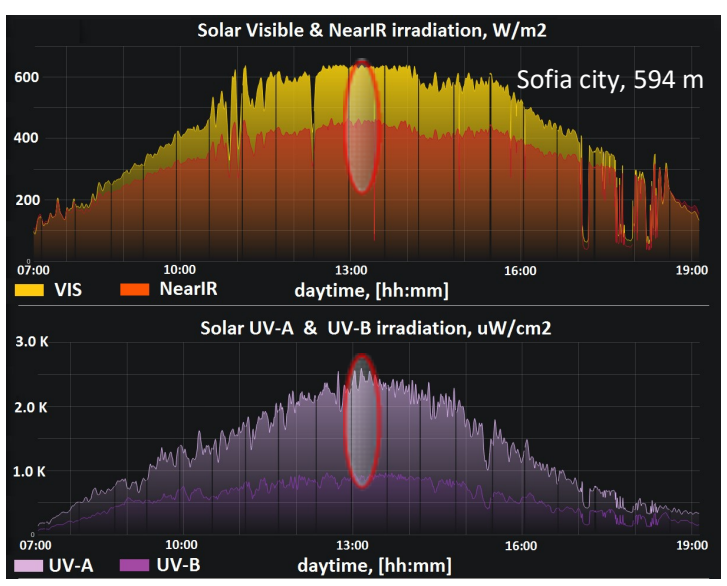

Figure 5. UV-A/UV-B/Vis/NIR daily profiles in an urban area and effect of pollution during a clear day with no traffic. (Red circles are indication for UV or Vis peak values)

The peak values of UV fluxes and Visible/NIR components differ in intensity and hour of the day. In rush hours (10:00-15:00) due to increased traffic pollution UV fluxes attenuate significantly (Fig.6).

\section{- Seasonal variation of Ozone concentration}

The intensity of solar UV-C and UV-B radiation is rather low, but the energy per photon is high. Due to this high energy, UV-C and UV-B radiation can have several harmful impacts on humans. Atmospheric gases absorb very little of the radiation at UV-A wavelengths, partially at UV-B and completely at UV-C which means that the variability is higher at smaller wavelengths. The cut-off of the solar spectrum in the UV-B is primarily a consequence of the sharp increase in the ozone absorption.

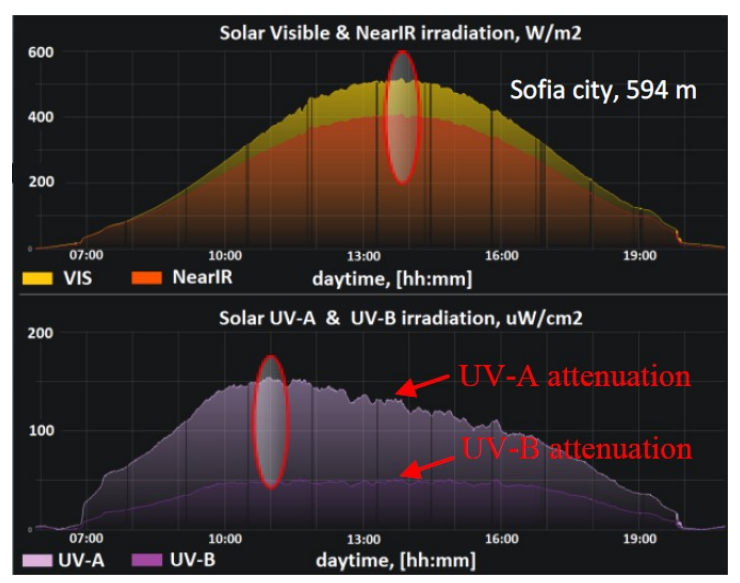

Figure 6. UV-A/UV-B/Vis/NIR daily profiles in an urban area and effect of pollution during a day with heavy traffic. (Red circles are indication for UV or Vis peak values)

At high altitudes it is expected the measurements to verify the inverse correlation between the ozone versus UVB levels. In fact, the observed daily maximum of ozone column amount is reached in March (355 DU) and daily minimum in October (270 DU) as can be seen at Fig. 7b. The maximum of UV-B is occurred in June and July period centered on the summer solstice. 
Typically at 27 of June the measured UV index is $>9$ (Fig 7a) [3].

The difference between the minimum of ozone amount and the maximum of UV-B, about 4 months earlier (in June, July) is likely associated with the stratospheric wind patterns, known as the BrewerDobson circulation $[8,9]$.

- Seasonal effect of elevated aerosols concentration

Air pollutants are generally efficient absorbers of UV radiation. Depending on their absorption capacity, aerosols can either reduce the UV irradiance reaching the Earth's surface or enhance it, especially the diffuse component through multiple scattering.

Recent studies [10] have shown that in urban areas, aerosols and air-pollutants such as tropospheric ozone and nitrogen dioxide can significantly attenuate solar UV radiation.

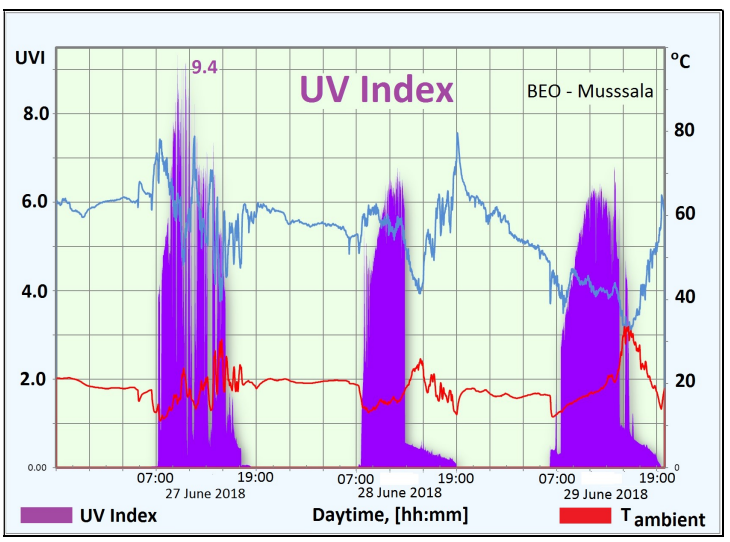

a) UVI maximum in June

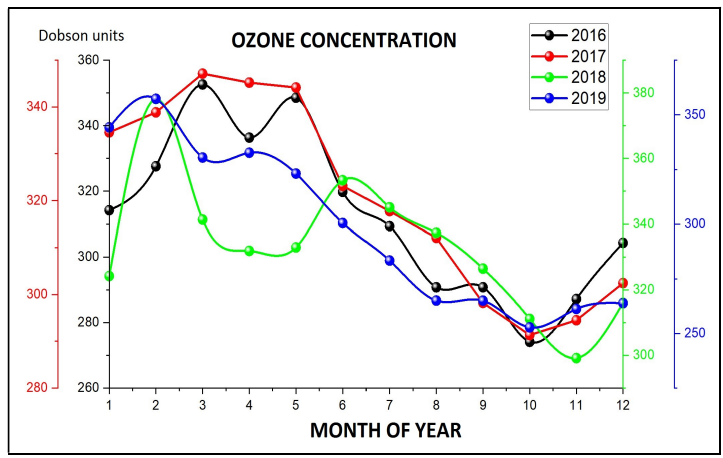

b) Ozone minimum in October

Figure 7. Seasonal variations of UV and Ozone concentration at BEO Moussala

Aerosols and trace gases from biomass burning can also affect the UV in a similar way. A clear confirmation of these findings can be seen in Figure 8 . Around noon hours in a winter month (November) the smoke (PM10 aerosol particles) of anthropogenic origin could affect on the daily profiles. The scattering and absorption of UV-A/UV-B fluxes differs significantly than Visible and NearIR components.

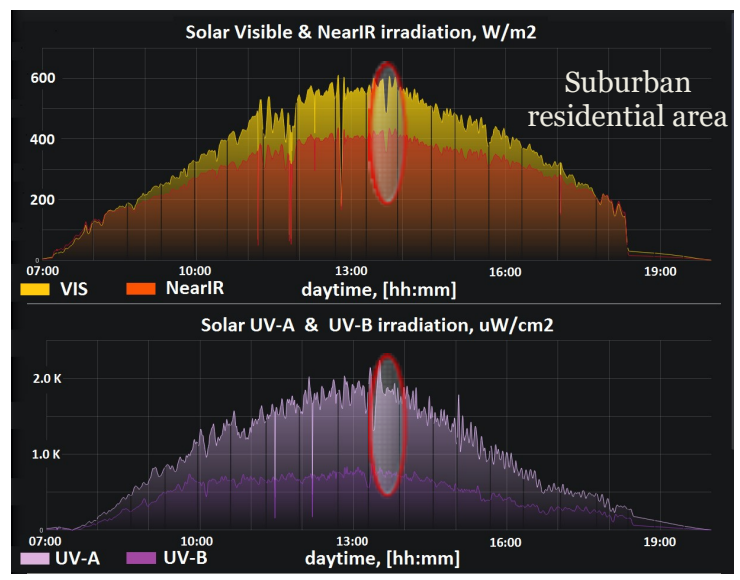

a) a day in September with high transparency

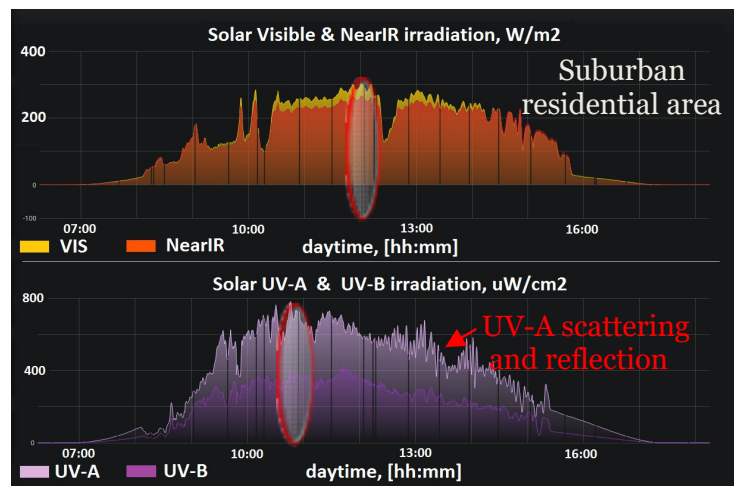

b) a day in November with smoke and aerosols

Figure 8. UV-A/UV-B/Vis/NIR daily profiles in a suburban location - effect of biomass burning (Red circles are indication for UV or Vis peak values)

\section{CONCLUSION}

An affordable datalogging and UV monitoring equipment has been built and installed in several locations in Bulgaria. It is based on open-source controllers and open-source software which satisfies the requirements in agricultural and economic sectors for real-time environmental monitoring and solar UV monitoring. It is easy to upgrade and is adaptable to different databases. Some first measurements with the new UV sensors system are presented. UV-A/UV-B radiant fluxes can be monitored and compared in order to prevent the population from overexposure or extraordinary solar events. Its feasibility lies in the usage of up-to-date hardware and open-source software that facilitate fast development of applications and measurements in Internet environment. The operator can access the installed UV sensor systems remotely at any time, visualize, and analyze graphs with data collected.

Acknowledgements: This study is supported by grants under projects No. KP o6 /24H - 2018 and No. DN 04/1 -2016 of National Research Fund of Bulgaria. 


\section{REFERENCES}

1. R. L. McKenzie, P. J. Aucamp, A. F. Bais, L. O. Björn, M. Ilyas, "Changes in biologically-active ultraviolet radiation reaching the Earth's surface," Photochem. Photobiol. Sci., vol. 6, pp. 218-231, 2007. https://doi.org/10.1039/b700017k

2. L. J. Gray et al., "Solar influences on climate," Rev. Geophys, vol. 48, no. 4, article no. RG4001, 2010. https://doi.org/10.1029/2009RG000282

3. N. Tyutyundzhiev, Ch. Angelov, K. Lovchinov, T. Arsov, H. Nichev, "Open-source tool for solar UV measurements," AIP Conference Proceedings, vol. 2075, no. 1, paper no. 130025, 2019. https://doi.org/10.1063/1.5091310

4. R. A. Atmoko, R. Riantini, M. K. Hasin, "IoT real time data acquisition using MQTT protocol," Journal of Physics: Conf. Series, vol. 853, article no. 012003, 2017. https://doi.org/10.1088/1742-6596/853/1/012003

5. Grafana 7.2.0., GrafanaLabs, 2020.

Retrieved from:

https://grafana.com/grafana/
6. G. Pfister, R. L. McKenzie, J. B. Liley, A. Thomas, B. W. Forgan, C. N. Long, "Cloud coverage based on allsky imaging and its impact on surface solar irradiance," J. Appl. Meteorol., vol. 42, no. 10, pp. 1421-1434, 2003. https://doi.org/10.1175/15200450(2003)042\%3C1421:CCBOAI\%3E2.O.CO;2

7. O. E. Malandraki, N. B. Crosby, "Solar Particle Radiation Storms Forecasting and Analysis," Astrophysics and Space Science Library, Springer (eBook), 2017.

https://doi.org/10.1007/978-3-319-60051-2

8. T. G. Shepherd, "Dynamics, stratospheric ozone, and climate change," Atmos.-Ocean, vol. 46, no. 1 , pp. 117-138, 2008. https://doi.org/10.3137/ao.460106

9. Q. Fu, P. Lin, S. Solomon, D. L. Hartmann, "Observational evidence of strengthening of the Brewer-Dobson circulation since 1980," J. Geophys. Res. Atmos., vol. 120, no. 19, pp. 10,214-10,228, 2015. https://doi.org/10.1002/2015JD023657

10. R. L. McKenzie et al., "Effects of urban pollution on UV spectral irradiances," Atmos. Chem. Phys. Discuss., vol. 8, no. 18, pp. 5683-5697, 2008. https://doi.org/10.5194/acp-8-5683-2008 